\title{
Essais
}

ESSAIS

Revue interdisciplinaire d'Humanités

$1 \mid 2012$

Varia

\section{« Migrants et dialectes » dans la presse italienne : représentations, enjeux et implications}

\section{Valeria Villa}

\section{(2) OpenEdition}

1 Journals

Édition électronique

URL : https://journals.openedition.org/essais/10648

DOI : 10.4000/essais. 10648

ISSN : 2276-0970

Éditeur

École doctorale Montaigne Humanités

Édition imprimée

Date de publication : 15 mai 2012

Pagination : 28-45

ISSN : 2417-4211

\section{Référence électronique}

Valeria Villa, « « Migrants et dialectes » dans la presse italienne : représentations, enjeux et

implications », Essais [En ligne], 1 | 2012, mis en ligne le 31 janvier 2022, consulté le 02 février 2022

URL : http://journals.openedition.org/essais/10648 ; DOI : https://doi.org/10.4000/essais. 10648 
Valeria Villa, Université Michel de Montaigne Bordeaux 3, EA 3656 AMERIBER et Università di Bologna (SITLEC), valeria. villa@u-bordeaux3.fr

\title{
Résumé
}

Cette contribution vise à montrer les représentations des immigrés véhiculées par la presse italienne au travers de la mise en valeur de leurs compétences linguistiques. Nous nous concentrons notamment sur la visibilité accordée par les médias - même par le biais de la polyphonie énonciative - à leur emploi et/ou connaissance des dialectes italiens, sur les implications dans leur intégration sociale et sur l'image de leurs pratiques linguistiques.

\section{Mots-clés}

migrants, dialectes italiens, presse, intégration sociale

\begin{abstract}
This paper aims to show immigrant representations portrayed by the Italian press by focusing on their linguistic competencies. We concentrated on the visibility given by the media - also by the means of enunciative polyphony - to their use and/or knowledge of Italian dialects, to the implications on their social integration and to the image of their linguistic practices.
\end{abstract}

\section{Keywords}

immigrants, Italian dialects, press, social integration 


\section{" Migrants et dialectes » dans la}

\section{presse italienne : représentations,}

\section{enjeux et implications}

\section{Introduction}

Les figures des migrants mises en scène par la presse écrite ont souvent été un objet d'étude pour de nombreuses raisons ${ }^{1}$. Il s'agit en effet de recherches qui se sont concentrées notamment sur l'immigration " prise aux mots $»^{2}$, sur les figures et les représentations médiatiques dans les pays d'accueil. Au niveau européen, elles ont mis en évidence, à plusieurs reprises, un portrait négatif des migrants : stéréotypé et qui repose sur ce que Binotto, Martino ${ }^{3}$ appellent le triangle " criminalité, clandestinité, arrivées ", des termes caractérisant les flux migratoires arrivant des pays du Sud et de l'Est de l'Europe.

En Italie, par exemple, les recherches sur la représentation des immigrés dans les médias ${ }^{4}$, pendant les vingt dernières années ont mis en avant la politisation du sujet, la mise en mots de l'altérité, les stéréotypes classiques caractérisant ce phénomène $e^{5}$ et récemment les discours sécuritaires. D’un côté nous avons des informations plus générales au sujet de l'immigration et des comportements

1. Cf. Teo Van Dijk, Critical News Analysis. Critical Studies, 1 (1), 1989, p. 103-126; Teo Van Dijk Teo, Racism and the press, New York, Routledge, 1991 ; Teo Van Dijk Teo, « New(s) Racism. A discourse analytical approach ", in Simon Cottle (sous la dir. de), Ethnic Minorities and the Media, Milton Keynes, Open University Press, 2000, p. 33-49.

2. Cf. Simone Bonnafous, L'immigration prise aux mots. Les immigrés dans la presse au tournant des années 80, Paris, Kimé, 1991.

3. Cf. Marco Binotto, Valentina Martino, Fuori luogo. L'immigrazione e i media italiani, Cosenza, Pellegrini editore, 2004, p. 31.

4. La recherche menée par le Censis a montré que les images des immigrés dans la télévision italienne résultent négatives dans 83 des cas sur 100, avec une majorité d'informations qui concernent la criminalité, l'assistance, la solidarité ou encore l'immigration comme sujet plus général ; de même, plusieurs séries télévisées montrent des représentations des migrants très simplifiées, Censis, Turning into diversity, immigrati e minoranze etniche nei media, $\mathrm{n}^{\circ} 1 / 2,2003$.

5. Cf. Marco Binotto, Valentina Martino, op. cit., p. 1 ; Mario Corte, Comunicazione e giornalismo interculturale. Pedagogia e ruolo dei mass madia in una società pluralistica, Padova, Cedam, 2006a ${ }^{\circ}$; Mario Corte, Stranieri e mass media, stampa immigrazione e pedagogia interculturale, Padova, Cedam, 2006b ; Pantaleone Sergi, Stampa migrante. Giornali della diaspora italiana e dell'immigrazione in Italia, Soveria, Rubettino Editore, 2010 ; Ribkall Sibathu, Il cittadino che non c'è. L'immigrazione nei media italiani, Roma, Edup, 2004. 
des immigrés; de l'autre on retrouve les attitudes et les craintes des italiens en lien avec la présence des étrangers, et enfin des nouvelles sur les débarquements, les arrivées et la dégradation qui en découle ${ }^{6}$.

Mais, parmi ces nombreuses images produites par la presse italienne, on découvre également une dimension qui sort de la représentation générale pour décrire un caractère plus spécifique : au travers des pratiques linguistiques, notamment, le rapport entre les migrants et la langue italienne, et plus récemment leur rapport avec les nombreux dialectes italiens ${ }^{7}$ (qui s'avèrent rester dans l'héritage et l'emploi, surtout oral, des locuteurs natifs).

Une remarque nécessaire concerne la visibilité accordée à ce thème dans la presse $^{8}$. Rendre visible, parler et " écrire " sur cet aspect signifie sans doute leur accorder une importance et de l'espace. Ce binôme - immigration-dialectes - constituera donc le focus de notre analyse linguistique. Avant de présenter cette dernière, nous procéderons d'abord en illustrant brièvement le corpus sélectionné, puis les principes méthodologiques choisis.

\section{Le corpus de l'enquête et les principes méthodologiques}

Les extraits sélectionnés et analysés dans cette étude ont été tirés de plusieurs journaux de la presse italienne. Parmi les quotidiens nationaux selectionnés on retrouve : la Repubblica, L'Espresso, il Corriere della Sera, la Stampa, il Giornale. Dans la catégorie de la presse régionale: Il Resto del Carlino, il Corriere del Veneto, La tribuna di Treviso etc. Les articles font partie d'un corpus plus large

6. Cf. Marco Binotto, Valentina Martino, op. cit., p. 39.

7. Cette situation linguistique très particulière qui se remarque surtout dans l'oralité voit les locuteurs dans des situations informelles se servir aussi bien de la variété haute (l'italien) que de celle basse (le dialecte), en situation d'alternance ou mixité codique. En revanche, dans la conversation formelle le locuteur dispose de l'italien standard. La définition plus adaptée pour cette situation linguistique semble être celle du linguiste Berruto (1987; 2006) qui la nomme " dilalie ». (Pour approfondir cette notion cf. Gaetano Berruto, "Lingua dialetto, diglossia, dilalia ", in G. Holtus-J. Kramer (sous la dir. de), Romania et Slavia adriatica, Amburgo, 1987, p. 57-81 ; Gaetano Berruto, "A mo' di introduzione », in A. Sobrero, A. Miglietta (sous la dir. de), Lingua e dialetto nell'Italia del duemila, Galatina, Congedo Editore, p. 5-13). En effet, la notion de diglossie de Ferguson n'est pas tout à fait adaptée au cadre italien, car il n'existe pas de séparation très nette d'emploi entre variété haute pour le contexte formel et basse pour le contexte informel, du moment que les deux peuvent coexister dans le deuxième. Cf. Charles Ferguson, "Diglossia », in Word 1, 1959, p. 325-340.

8. Néanmoins, l'emploi même du dialecte dans la presse italienne est toujours un sujet d'actualité comme démontrent des recherches sur les dialectes dans les journaux de Palisi. Cf. Ida Palisa, "Il dialetto nei giornali », in De Blasi Nicola, Marcato Carla (sous la dir. de), Lo spazio del dialetto in città, Liguori, Napoli, 2006, p. 113-142. 
« Migrants et dialectes » dans la presse italienne :

représentations, enjeux et implications

qui accueille également du matériel saisi en ligne (publicités, vidéos etc.) et des œuvres de la littérature de l'immigration?.

Nous avons retenu la période 2008-2011, en constituant un corpus de 40 articles quantitativement modeste, mais qualitativement significatif, car les articles parus en ligne abordent le binôme immigration-dialectes, de différents points de vue et dans des contextes variés. Les passages, une fois classés par date et source et selon les thèmes liés aux variétés vernaculaires qu'ils traitent, ont été analysés selon le cadre théorique de l'analyse des textes de communication ${ }^{10}$.

Pour ce qui est des cas examinés dans cette contribution, les passages ont été divisés en deux types : d'une part ceux qui mettent en évidence la capacité des migrants à parler en dialecte (cf. 3) où l'énonciateur correspond toujours au journaliste ; d'autre part ceux qui, pour le même but, font en revanche appel au discours direct (dorénavant DD) et à différents énonciateurs (journalistes, citoyens natifs, migrants) (cf. 4). Ils ont été étudiés en tenant compte de la notion de polyphonie ${ }^{11}$ énonciative ${ }^{12}$ notamment dans les discours de la presse ${ }^{13}$.

\section{Le rapport des migrants au dialecte dans la presse}

D'après une macro-catégorisation qui découle d'une première lecture qui met en évidence la manière dont les articles se référent le plus souvent à :

\section{a. La volonté politique de mettre en place des cours de dialecte pour les migrants}

De nombreux articles mettent en lumière la nécessité, selon le parti de droite de la Ligue du Nord, pour les migrants travaillant dans le nord de

9. Dans cette contribution nous nous concentrerons exclusivement sur la presse. Le thème dialectes-immigration dans les médias, films et littérature est approfondi dans le cadre de notre thèse de doctorat.

10. Cf. Dominique Maingueneau, Analyser les textes de communication, Paris, Arman Colin, 2007.

11. Le discours rapporté intégré dans les théories de la polyphonie énonciative est clairement un concept très complexe et un domaine assez vaste. Les titres indiqués ici ne reflètent qu'une partie réduite des recherches à ce sujet. Par conséquent, dans ce travail, nous ferons référence notamment aux auteurs sous-cités (cf. 2). En outre, il est important de souligner que nous ne rentrerons pas dans la discussion concernant la fidélité du discours rapporté.

12. Cf. Mikhail Bakhtine, Esthétique et théorie du roman, Paris, Gallimard, 1978 ; Mikhail Bakhtine, La Poétique de Dostoievski, Paris, Seuil, 1963 ; Oswald Ducrot, Le Dire et le dit, Paris, Minuit, 1984 ; Perrin L., "Polyphonie et autres formes d'hétérogénéité énonciative : Bakhtine, Bally, Ducrot ", Pratiques, n 123-124, 2004, p. 3-7 ; André Petitjean (sous la dir. de), "Polyphonie », Pratiques n 123-124, 2004 ; André Petitjean, « Les faits divers : polyphonie énonciative et hétérogénéité textuelle ", Langue française, ${ }^{\circ}$ 74, 1987, p. 73-96.

13. Cf. Greta Komur, "Les modes du discours rapporté dans la presse et leurs enjeux polyphoniques ", Pratiques, n 123-124, 2004, p. 57-74. 
l'Italie d'apprendre les dialectes locaux, et, pour ce faire, ils proposent de créer des cours de dialecte ; il s'agit souvent de cours proposés aux femmes ("le badanti») qui s'occupent des personnes âgées ou encore aux migrants employés dans les chantiers comme maçons. Dans ces deux milieux l'emploi du dialecte étant très marqué, ces articles veulent démontrer que la maîtrise ${ }^{14}$ de la variété vernaculaire est indispensable. Dans cette optique, il y a aussi des propositions sur l'évaluation des compétences linguistiques en italien mais aussi de la connaissance de la culture locale.

\section{b. La volonté des migrants eux-mêmes d'apprendre le dialecte, ou l'aversion à fréquenter un cours de dialecte}

De la part des migrants, lorsque les journalistes leur donnent la possibilité de s'exprimer à ce propos, on retrouve des attitudes controversées : à côté de migrants qui n'apprécieraient pas de fréquenter des cours de dialecte, d'autres au contraire les réclament personnellement, ou encore les fréquentent déjà.

\section{c. Leur emploi du dialecte dans différents contextes et le rapport à l'intégration}

On retrouve dans certains articles des remarques concernant simplement la capacité des migrants à parler un dialecte (cf. exemples 1, 2 de l'analyse), les fonctions attribuées à ces pratiques, comme dans le cas de l'emploi du dialecte pour des raisons conflictuelles ${ }^{15}$ ou bien comme ressource transactionnelle

14. Voici un exemple d'un des articles abordant ce sujet, suivi de la traduction : "Vorìa magnare capùssi, pisacan, formaio. Non me sento ben : go la mossa... ». Come potrebbe reagire secondo voi, una badante proveniente dalla Moldavia di fronte ad espressioni che non ricalcano l'italiano forbito ? Eppure molti anziani residenti in città e in provincia parlano così abitualmente, soprattutto se hanno superato gli ottanta [...]. Come correre ai ripari di fronte ad un dialetto che rimane spesso la lingua principale per i veneti ? Con dei corsi di dialetto [...]. Da una giunta leghista la scelta del dialetto forse può apparire scontata. Ma in questo caso non si parla di scuole elementari o medie. Si parla di anziani e di donne che si occupano di loro : pianeti diversi, mondi lontani, usanze differenti. Il giornale di Vicenza, 13.01.2011. Trad. "Je voudrais manger du chou-fleur, des légumes et du fromage. Je ne suis pas bien... ». Comment pourrait-elle réagir d'après vous, une aide soignante arrivant de la Moldavie devant des expressions qui ne respectent pas l'italien standard ? Toutefois, plusieurs personnes âgées parlent de cette façon habituellement, surtout s'ils ont plus que 80 ans ? Comment faire face au dialecte qui demeure la langue principale pour les vénitiens ? Avec des cours de dialecte [...]. Le choix du dialecte n'étonnera personne venant d'un conseil municipal de la Ligue Nord. Mais en ce cas spécifique, on ne parle ni d'écoles primaires, ni de collèges. On parle de personnes âgées et des femmes qui s'en occupent : planètes différentes, mondes lointains, coutumes différentes.

15. Sur l'emploi du dialecte pour des fonctions conflictuelles cf. Valeria Villa, "La communication interculturelle entre migrants et italiens : choix linguistiques d'exclusion et d'inclusion ", in M. Lebon-Eyquem, T. Bulot, G. Ledegen, (sous la dir. de) Ségrégation, normes 
« Migrants et dialectes » dans la presse italienne :

représentations, enjeux et implications

etc. ; d'autres articles vont plus en profondeur sur ce phénomène en le mettant en rapport avec l'intégration sociale (cf. exemples 3, 4, 5, 6, 7).

\section{d. Le problème de compréhension ${ }^{16}$ des dialectes}

D’un nombre plus réduit, ces articles montrent la difficulté pour les migrants de comprendre certains dialectes.

Dans cette contribution, nous aborderons de façon détaillée le point " $\mathrm{c}$ ». En effet, dans plusieurs articles, l'un des indices les plus frappants concerne des phrases où les énonciateurs mettent en évidence la compétence dialectale des immigrés (cf. 3).

Cette analyse vise donc à déceler les raisons de ces mises en relief et les valeurs attachées par les natifs ou les migrants à la connaissance du dialecte. Les journalistes nous donnent des informations à ce sujet, à travers la polyphonie énonciative, c'est-à-dire la superposition de voix dans le même passage, en montrant les différents énonciateurs qui commentent les conduites communicatives des migrants dans leur emploi du dialecte (cf. 4).

\section{3. « Les migrants parlent aussi les dialectes "}

Nous commencerons par les différents procédés mis en œuvre par les énonciateurs pour souligner la connaissance du dialecte par les immigrés. Dans le corpus analysé, nous avons retenu 15 articles sur 40 (soit 37.5\%), abordant des sujets variés, qui mettent en évidence ce phénomène : «les migrants parlent aussi le dialecte " à travers des modalités diverses.

Nous les avons classés par source (et date) et par dialecte parlé par les migrants (en prenant en compte les verbes employés pour le mettre en évidence). Ensuite, on retrouve (cf. T.1) un résumé très bref à propos du sujet de l'article et enfin les termes employés dans les passages sélectionnés pour représenter les agents (migrants). Souvent, il s'agit de la nationalité, parfois des prénoms, de l'étiquette d'étranger et dans un cas de la couleur de la peau. Par conséquent, il peut y avoir aussi des exemples qui risquent de montrer des images stéréotypées. Ces catégories représentatives soulignent une habitude des journaux italiens, qui consiste à ne désigner les citoyens étrangers qu'à travers la nationalité d'origine, ou tout simplement à travers la qualification d' "immigré " (parfois sans papiers), cette désignation étant la seule information personnelle sur le(s)

et discrimination(s), Sociolinguistique urbaine et migrance, Journées internationales de sociolinguistique urbaine, Eme, Bruxelles, 2012.

16. Dans un cas isolé, deux quotidiens reportent un épisode de violence subi par un migrant sénégalais, battu par un natif car il ne comprenait pas ce qu'on lui disait en dialecte de Trévise. (Rompe una gamba a operaio senegalese : "Non capiva il dialetto veneto ", tiré de il Messaggero, 31.07.2011 et Non parli il dialetto? Allora ti do' una lezione e il giustiziere veneto massacra il senegalese, tire de il Giornale, 31.07.2011). 
protagoniste(s) des faits rapportés ${ }^{17}$. Cela met en lumière les limites des mass media par rapport à un sujet sensible comme celui de l'immigration, et met en exergue les difficultés liées à la gestion de la diversité culturelle.

Une fois ces critères de sélection posés, nous les avons synthétisés de façon schématique dans le tableau (T.1.) :

\begin{tabular}{|c|c|c|c|}
\hline SOURCE & DIALECTE & À PROPOS DE/DU/DES & DÉNOMINATIONS DES AGENTS \\
\hline \multicolumn{4}{|c|}{ Il corriere della sera } \\
\hline 1) 21.08 .2009 & $\begin{array}{l}\text { "parler plus } \\
\text { dialecte que } \\
\text { italien" }\end{array}$ & $\begin{array}{l}\text { L'équipe nationale de } \\
\text { cricket }\end{array}$ & $\begin{array}{l}\text { "enfants d'émigrés "; } \\
\text { Plusieurs nationalités }\end{array}$ \\
\hline 2) 06.06 .2009 & $\begin{array}{l}\text { dialecte de Voghera } \\
\text { "parler" }\end{array}$ & $\begin{array}{l}\text { Concours de poésie dans } \\
\text { une école primaire }\end{array}$ & Prénom « Lihao »; « chinois »; \\
\hline 3) 30.08 .2010 & $\begin{array}{l}\text { dialecte de } \\
\text { Sant'Angelo; } \\
\text { "dire bonjour en } \\
\text { dialecte" }\end{array}$ & $\begin{array}{l}\text { Cours de dialecte local } \\
\text { pour les migrants }\end{array}$ & $\begin{array}{l}\text { "vendeur ambulant "; référence à } \\
\text { la couleur de la peau ; }\end{array}$ \\
\hline 4) 10.05 .2010 & $\begin{array}{l}\text { dialecte de } \\
\text { Padoue ; "parler } \\
\text { parfaitement " }\end{array}$ & $\begin{array}{l}\text { Histoires de jeunes } \\
\text { immigrés de première } \\
\text { génération }\end{array}$ & $\begin{array}{l}\text { « italien "; " marocain "; } \\
\text { « Mohammed "; « ses amis "; }\end{array}$ \\
\hline 5) 03.02 .2010 & $\begin{array}{l}\text { dialecte vénitien ; } \\
\text { "capables de parler } \\
\text { dialecte" }\end{array}$ & $\begin{array}{l}\text { La statistique sur } \\
\text { la connaissance du } \\
\text { dialecte vénitien de la } \\
\text { part d'étudiants et de } \\
\text { travailleurs immigrés }\end{array}$ & $\begin{array}{l}\text { " étrangers »; « immigrés "; } \\
\text { " nouveaux vénitiens " }\end{array}$ \\
\hline \multicolumn{4}{|c|}{ La repubblica } \\
\hline 6) 07.03 .2011 & $\begin{array}{l}\text { dialecte sicilien- } \\
\text { marocain ; " hurler } \\
\text { en dialecte" }\end{array}$ & $\begin{array}{l}\text { Évolutions récentes des } \\
\text { familles et des mères } \\
\text { italiennes }\end{array}$ & $\begin{array}{l}\text { "vendeur ambulant"; } \\
\text { " marocain "; " pauvre "; " mal } \\
\text { habillé "; " sans dentition" }\end{array}$ \\
\hline 7) 21.09 .2008 & $\begin{array}{l}\text { dialecte napolitain ; } \\
\text { " expliquer en } \\
\text { dialecte" }\end{array}$ & $\begin{array}{l}\text { Migrants qui demandent } \\
\text { plus de droits }\end{array}$ & $\begin{array}{l}\text { Prénom " Isaac "; « victimes »; } \\
\text { " jeune immigré "; }\end{array}$ \\
\hline 8) 06.04 .2010 & $\begin{array}{l}\text { dialecte vénitien ; } \\
\text { "menacer en } \\
\text { dialecte" }\end{array}$ & $\begin{array}{l}\text { Femmes au pouvoir dans } \\
\text { la ligue du Nord : un } \\
\text { livre les raconte }\end{array}$ & "immigré "; « tunisien » \\
\hline 9) 05.05 .2011 & $\begin{array}{l}\text { dialecte de Parme } \\
\text { "parler» }\end{array}$ & $\begin{array}{l}\text { Données quantitatives } \\
\text { actuelles et futures des } \\
\text { immigrées à Parme }\end{array}$ & $\begin{array}{l}\text { "sénégalais "; " tout à fait } \\
\text { italiens" }\end{array}$ \\
\hline \multicolumn{4}{|c|}{ Corriere del veneto } \\
\hline
\end{tabular}

17. Cf. Marco Binotto, Valentina Martino, op. cit. p. 1. 
« Migrants et dialectes » dans la presse italienne :

représentations, enjeux et implications

\begin{tabular}{|c|c|c|c|}
\hline 10) 18.09 .2009 & $\begin{array}{l}\text { dialecte véronais; } \\
\text { "parler"; } \\
\text { "apprendre" }\end{array}$ & $\begin{array}{l}\text { Une famille chinoise } \\
\text { rachète un bar du } \\
\text { centre-ville de Vérone }\end{array}$ & Prénoms « John »; " Feng Feng " \\
\hline 11) 28.07 .2011 & $\begin{array}{l}\text { dialecte vénitien ; } \\
\text { "capables de } \\
\text { parler"; }\end{array}$ & $\begin{array}{l}\text { Statistiques sur les } \\
\text { nouveau-nés de la } \\
\text { Vénétie, } 1 / 3 \text { a au moins } \\
\text { un parent étranger }\end{array}$ & $\begin{array}{l}\text { " origine non italienne"; } \\
\text { "travailleurs étrangers"; }\end{array}$ \\
\hline \multicolumn{4}{|c|}{ Il giornale } \\
\hline 12) 26.07 .2009 & $\begin{array}{l}\text { dialecte napolitain } \\
\text { " connaître "; }\end{array}$ & $\begin{array}{l}\text { Un jeune japonais qui } \\
\text { vient en Italie pour } \\
\text { apprendre le métier de } \\
\text { pizzaiolo }\end{array}$ & $\begin{array}{l}\text { Prénom « Makoto " } \\
\text { «japonais » }\end{array}$ \\
\hline 13) 24.03 .2010 & $\begin{array}{l}\text { Dialecte } \\
\text { (générique); } \\
\text { "parler»; }\end{array}$ & $\begin{array}{l}\text { Un politicien de droite } \\
\text { propose la naturalisation } \\
\text { pour les enfants } \\
\text { d'immigrés nés en Italie }\end{array}$ & $\begin{array}{l}\text { "les enfants des immigrés"; } \\
\text { " créatures"; }\end{array}$ \\
\hline \multicolumn{4}{|c|}{ Il resto del carlino } \\
\hline 14) 03.12 .2009 & $\begin{array}{l}\text { dialecte vénitien ; } \\
\text { dialecte napolitain ; } \\
\text { dialecte } \\
\text { piémontais ; } \\
\text { "parler"; }\end{array}$ & $\begin{array}{l}\text { Cas du footballeur } \\
\text { Ballotelli (de couleur) } \\
\text { qui est le symbole du } \\
\text { passage de l'Italie de hier } \\
\text { à celle actuelle }\end{array}$ & $\begin{array}{l}\text { "garçons d'origine africaine " } \\
\text { "poussins" }\end{array}$ \\
\hline \multicolumn{4}{|c|}{ L'espresso } \\
\hline 15) 26.04 .2010 & $\begin{array}{l}\text { dialecte calabrais; } \\
\text { dialecte véronais ; } \\
\text { "parler»; } \\
\text { "s'exprimer»; }\end{array}$ & $\begin{array}{l}\text { Premier congrès national } \\
\text { des immigrés à Rome }\end{array}$ & $\begin{array}{l}\text { Prénom « Oxana »; la " Tahar ", } \\
\text { " la fille "; « ukrainienne "; } \\
\text { " tunisien »; }\end{array}$ \\
\hline
\end{tabular}

\section{Tableau 1. Dialectes et représentations des immigrés} dans les quotidiens italiens

Le tableau nous aidera à visualiser les dialectes principaux concernés par les articles, leurs sujets, et surtout les représentations des migrants.

Dans cette optique, nous examinerons les extraits les plus représentatifs de façon détaillée, en commençant par trois cas d'étude tirés du Corriere della Sera, Corriere del Veneto et l'Espresso. Ici nous pouvons saisir des références à l'emploi du dialecte et cette thématique nous amène à réfléchir à son rapport à l'intégration.

L'extrait $\mathrm{n}^{\circ} 1$ (cf. T. $1 \mathrm{n}^{\circ} 11$ ) montre que parmi les futurs vénitiens il y aura également des migrants. 
1. E se tra meno di vent'anni i veneti potrebbero dunque essere per un terzo uomini e donne di origine non italiana - capaci comunque di parlare dialetto - oggi i lavoratori stranieri sono 230 mila, poco più del 10 per cento della popolazione attiva totale ${ }^{18}$. (Corriere del Veneto, 28.07.2011)

Il s'agira d'hommes et de femmes "d'origine non italienne" qui, indépendamment de leur origine étrangère, seront capables "de toute façon " de parler le dialecte, comme l'incise isolée par des tirets nous le signale. Nous pourrions présumer que ce supplément d'information mis en relief est employé pour mettre en avant une qualité positive attribuée aux migrants, qualité qui servira probablement à leur intégration sociale et linguistique dans la région. Il faut souligner que le dialecte de la Vénétie est souvent objet de revendications politiques par le parti de la Ligue du Nord dans les médias, à cause surtout d'une vitalité très importante de ce dialecte.

Le partage du dialecte pourrait faire partie d'une image d'immigré intégré qui connaît la culture et aussi la langue locale. De la mise en scène de l'altérité résulte donc une ambivalence : étranger mais "capable» de parler le dialecte. Ce mais adversatif résonne en effet de façon très significative dans ce contexte, comme nous le montre une analyse spécifique menée par Rigouste ${ }^{19}$ sur la représentation de l'immigration maghrébine dans la presse française, dans laquelle il montre que les « figures de l'Ami et de l'Ennemi » sont strictement liées.

Ensuite, deux autres remarques linguistiques s'imposent pour ce qui est du portrait des migrants : la référence nuancée à la provenance étrangère et non complètement italienne, et surtout la représentation quantitative ("1/3, 230 mille, $10 \% »)$. Cette dernière signale l'augmentation démographique actuelle et future dans la région de la Vénétie. Ce dernier aspect quantitatif est très délicat dans les medias, car très souvent les informations sur l'immigration relèvent du "syndrome de l'invasion ${ }^{20}$.

L'exemple $\mathrm{n}^{\circ} 2$ se présente, en revanche dans un rapport différent à l'insertion sociale et linguistique (cf. T.1 $\left.\mathrm{n}^{\circ} 15\right)$. Ici, le domaine d'emploi de la variété

18. «Et si dans moins de vingt ans les vénitiens pourraient donc être pour un tiers des hommes et des femmes d'origine non italienne - capables de toute façon de parler dialecte - aujourd'hui les travailleurs étrangers sont 230 mille, un peu plus de $10 \%$ de toute la population active."

19. Mathieu Rigouste, «Les « jeunes issus de l'immigration » dans la presse écrite. Sur la théâtralisation médiatique d'une altérité politique », in Rencontres Jeunes et société http://www. jeunes-et-societes.com/public/archive2005.html, 2005.

20. Marco Bruno, «L'ennesimo sbarco di clandestini : la tematica degli arrivi nella comunicazione italiana », in M. Binotto, V. Martino (sous la dir. de), Fuori luogo. L'immigrazione e $i$ media italiani, Cosenza, Pellegrini editore, 2004, p. 105-106. 
«Migrants et dialectes » dans la presse italienne :

représentations, enjeux et implications

vernaculaire ne paraît pas réservé qu'à l'interaction avec les natifs. En effet, le journaliste met en évidence une pratique assez particulière caractérisant la conversation entre des migrants d'origines différentes : la langue véhiculaire est l'italien mais aussi le dialecte. Ensuite, il remarque l'opposition entre la nationalité des migrants, une Ukrainienne et un Tunisien et le dialecte qu'ils parlent, le calabrais et le véronais. Nous n'avons pas d'indications précises sur l'âge des protagonistes, mais pour Oxana, l'Ukrainienne, l'énonciateur parle d'une "fille" et cela nous amène à préciser que l'emploi du dialecte dans la langue des jeunes est un comportement linguistique fréquent pour de nombreuses raisons ${ }^{21}$.

2. Nei tavoli si parla di scuola, famiglia, tasse, permessi, sindacato e di delusioni, tutti in cerchio. La lingua franca è l'italiano ma spesso anche il dialetto: Oxana, la ragazza che si occupa dei temi femminili, è ucraina e parla calabrese, Tahar è tunisino e spesso si esprime in veronese. Si parla di politica, in un modo diverso da quello che si vede in tv, il tempo è poco e il momento difficile ${ }^{22}$ (L'Espresso, 26.04.2010).

Ce qui est intéressant ici est l'emploi de la coordination (ukrainienne et parle calabrais) entre l'origine géographique très éloignée et la proximité constituée par la connaissance de la langue locale : ces données ne sont pas représentées linguistiquement à travers un adverbe d'opposition mais à travers une conjonction. Ces dispositifs linguistiques pourraient désigner une coexistence possible ou encore l'étrangeté du phénomène.

Cela dit, dans les deux cas, cette mise en relief est opérée avec des adverbes ("de toute façon" et "aussi ») qui nous donnent des indices sur la mise en valeur de cette pratique linguistique des migrants par les journalistes. Ces derniers montrent comment leur connaissance du tissu socio-local fait écho, de façon implicite, au concept d'intégration. Donc parler un dialecte peut signifier : connaître et avoir intégré les valeurs du territoire où les étrangers vivent et travaillent.

21. Cf. Michele Cortellazzo, "Il parlato giovanile», in L. Serianni, P. Trifone (sous la dir. de), Storia della lingua italiana II. Scritto e parlato, 1990, p. 291-317 ; Carla Marcato, Dialetto, Dialetti, Italiano, Bologna, Il Mulino, 2002, p. 43-55.

22. Dans les tables (rondes) on parle d'école, de famille, d'impôts, de permis, de syndicats et de déceptions tous en cercle. La langue véhiculaire est l'italien mais aussi le dialecte : Oxana, la fille qui s'occupe des thèmes féminins, est ukrainienne et parle calabrais, Tahar est tunisien et souvent il s'exprime en véronais. On parle de politique, d'une façon différente par rapport à ce qu'on voit à la télé, le temps est limité et le moment difficile. 
Après avoir examiné les deux cas qui ne relèvent pas du DD nous nous dirigerons vers les exemples qui se servent de la polyphonie énonciative avec des buts variés.

\section{Les représentations médiatiques à travers la polyphonie énonciative}

À travers une lecture attentive de ces articles on remarque que plusieurs énonciateurs nous rendent compte des nouvelles sur le sujet du dialecte et des migrants. Caractérisées par la polyphonie énonciative, ces nouvelles sont exprimées par une multiplicité de voix dans et à propos du sujet abordé, lorsque le journaliste donne la parole à d'autres interlocuteurs à l'aide du discours direct ${ }^{23}$. Celle du DD paraît la pratique de plus en plus exploitée par le journaliste contemporain qui est "souvent moins quelqu'un qui décrit souverainement le monde de l'extérieur que quelqu'un qui privilégie l'empathie, qui cherche à faire percevoir au lecteur le monde à travers les yeux des personnages qu'il évoque $»^{24}$.

Dans les 15 cas des fragments sélectionnés (T.1), on remarque une parité des pourcentages des responsabilités énonciatives, les trois énonciateurs (journalistes, natifs, migrants) sont également représentés. En revanche, parmi les dix fragments analysés, la responsabilité de ces commentaires est attribuée aux journalistes dans les extraits $1 ; 2 ; 7 ; 10$, aux migrants dans les exemples 5 et 9 et enfin à d'autres citoyens natifs dans les fragments $3 ; 4 ; 6 ; 8$; ils commentent tous les pratiques langagières des immigrés. On verra comment cette particularité se présente dans les passages examinés et affecte les propos reportés à travers la voix des plusieurs énonciateurs.

\subsection{Dialecte : valeur ajoutée et signe d'intégration sociale?}

Les exemples que nous étudierons dans cette section relèvent tous du DD et ici nous observons la présence de plusieurs énonciateurs. Pour cette présence, plusieurs dispositifs sont exploités afin de mettre en lumière la connaissance du dialecte, entre-autre le résumé avec citations, c'est-à-dire, " un résumé d'un texte dont l'original apparaît par fragments dans le fil du discours. Ce procédé facilite la distinction entre les paroles du journaliste et celles citées ${ }^{25}$. C'est le cas notamment de l'exemple $\mathrm{n}^{\circ} 3$ (cf. T. $1 \mathrm{n}^{\circ} 2$ ) où l'on retrouve Lilhao, un enfant chinois, ayant gagné un concours de poésie dialectale organisé par son école primaire. À ce propos les adultes cités dans l'article et Lihao lui-même commentent les faits et ils sont appelés en cause dans le passage à travers une forme assez hybride qui mélange deux énonciateurs dans le DD sous forme d'un résumé avec citations.

23. Cf. Mainguenau, op. cit. p. 3 et Komur, op. cit. p. 3.

24. Cf. Mainguenau, ibid., p. 138.

25. Ibid., p. 135-136. 
« Migrants et dialectes » dans la presse italienne :

représentations, enjeux et implications

3. «La poesia ? L'ho scritta in un pomeriggio ». Tutta opera tua ? "Proprio tutta no» (a). "Per forza - intervengono i grandi - un conto è parlare il dialetto, e Lihao lo parla, un conto è scrivere : è difficile anche per noi...» (b) Una legittima consulenza, insomma. E da grande? "Poliziotto. E anche agente di viaggio " ${ }^{26}$ (c) (Il Corriere della Sera, 06.06.2009)

Le premier segment présenté (a) est attribué à Lihao qui dit, dans un dialogue avec le journaliste probablement, ne pas avoir écrit tout seul la poésie. Cependant on ne retrouve pas de segment présentateur, ce qui nous amène à penser qu'il pourrait s'agir d'un DD déguisé. La seule référence précise est située dans l'incise médiane ( "les adultes interviennent ») précédée et suivie par des tirets, qui nous montre l'identité d'un deuxième énonciateur des segments présentés : les adultes. Ces derniers commentent les compétences dialectales du jeune chinois. En effet, dans ce passage, on découvre qu'il a été aidé par ses enseignants dans la rédaction des vers, puisque la compétence écrite du dialecte est bien plus compliquée que la compétence orale même pour les adultes. Et cette dernière, possédée par Lilhao, est mise en évidence à travers, l'incise " et Lihao le parle " qui soustrait ce segment afin de spécifier, à travers les paroles des adultes interviewés, que le jeune chinois connaît et parle le dialecte local. Le dernier énoncé (c), privé du segment présentateur, semblerait attribué également au jeune chinois ; ici le segment présenté (" policier et aussi agent de voyage ") répond en effet directement à la question du journaliste sur les projets futurs de Lihao. Dans ce fragment, on remarque immédiatement les caractéristiques du " dialogisme ${ }^{27}$, plusieurs voix sont interpellées au sujet de Lihao, y compris le jeune chinois, avec un résultat de grande promptitude.

En revenant au sujet du dialecte, nous avons donc l'impression que la capacité à employer et à connaître une variété vernaculaire s'avère une caractéristique positive chez les migrants, assez pertinente pour être mise en évidence par la presse. Dans le cas de Lihao cela est très évident, un jeune chinois qui gagne un concours de poésie dialectale est une nouvelle étonnante et qui paraît assez insolite pour les natifs. Nous avons remarqué souvent cet effet de surprise et d'étonnement dans les articles du corpus. Cela se révèle particulièrement intéressant si l'énonciateur correspond à un natif autre que le journaliste, comme dans l'extrait suivant (cf. T1 $\left.\mathrm{n}^{\circ} 14\right)$ :

26. "La poésie ? Je l'ai écrite en un après-midi ». Tu l'as fait tout seul ? " Pas vraiment tout seul ". "Forcement - commentent les adultes - une chose est parler le dialecte, et Lihao le parle, une chose est l'écrire : c'est difficile même pour nous... » une aide légitime, donc. Et quand tu seras plus grand ? "Policier. Ou même agent de voyage ".

27. Cf. Bachtine, op. cit., p. 3, note 11 . 
4. Ha spiegato a La Stampa Franco Rossi, uno che organizza tornei giovanili di calcio, che l'Italia è piena di "pulcini » come superMario (a) : "Ti accorgi che quei ragazzi di origine africana sono in realtà italianissimi quando li senti parlare con i compagni in dialetto veneto, romano, napoletano, piemontese... ${ }^{28}(\mathrm{~b})$. (Il Resto del Carlino, 01.12.2009)

On retrouve ici une forme hybride qui résulte de la fusion du discours indirect (a) et du discours direct (b). Le premier énoncé (a) met en évidence que la responsabilité énonciative est confiée à Franco Rossi, un organisateur de tournois de football. Nous remarquons cet aspect également dans le procédé de mise entre guillemets dans l'emploi de l'appellatif « les poussins ", désignant de très jeunes footballeurs, pour "renvoyer la responsabilité à un autre " ${ }^{29}$ le discours indirect faisant référence aux propos tenus par l'énonciateur. Ensuite, le segment présentateur du discours indirect qui introduit également le DD, met en avant que ces garçons s'expriment aussi en parlant les dialectes locaux.

Un adjectif intéressant "italianissimi ", c'est-à-dire "tout à fait italiens ", concourt à la représentation des jeunes étrangers. Il montrerait que l'origine africaine de ces jeunes footballeurs ne met pas en question leur appartenance locale et linguistique aux régions italiennes de résidence, témoignée par la compétence dialectale en vénitien, romain, napolitain ou piémontais qu'ils parlent avec leurs camarades. Dans le cas présenté la compétence dialectale semble apparaître comme un indice "d'italianité ».

Des propos similaires sont tenus aussi dans l'exemple $\mathrm{n}^{\circ} 5$ (cf. T. $1 \mathrm{n}^{\circ}$ 9). En revanche, dans ce cas, il faudra remarquer qu'il ne s'agit pas d'un natif qui commente les pratiques linguistiques des étrangers. Le responsable énonciatif, en effet, est un migrant qui donne personnellement son avis au sujet de l'emploi du dialecte à travers le $\mathrm{DD}$.

5. I nostri figli sono nati qui e si sentono italianissimi - spiega il senegalese al pubblico - molti di loro parlano anche in dialetto parmigiano $^{30}$. (La Repubblica, 05.05.2011)

28. Franco Rossi, à une personne qui organise des tournois de football pour les jeunes, a expliqué au quotidien la Stampa que l'Italie est pleine de "poussins » comme Super- Mario (a) : "Tu t’aperçois que ces garçons d'origine africaine sont en réalité tout à fait italiens quand tu les entends parler avec leurs camarades en dialecte vénitien, romain, napolitain, piémontais... » (b).

29. Mainguenau, op. cit., p. 3.

30. Nos enfants sont nés ici et ils se sentent tout à fait italiens - le sénégalais explique-t-il au public - beaucoup d'entre eux parlent également le dialecte parmesan. 
« Migrants et dialectes » dans la presse italienne :

représentations, enjeux et implications

Même si dans cet énoncé on remarque l'absence des guillemets, cela ne nous empêche pas de le reconnaître comme un DD, car les indications sur l'énonciateur sont situées dans l'incise médiane isolée par des tirets. On apprend ainsi qu'il s'agit d'un homme d'origine sénégalaise. Comme nous l'avons souligné dans le paragraphe 3, et remarqué dans plusieurs passages, le recours à la nationalité pour la désignation du migrant est une pratique fréquente dans la presse. L'énonciateur s'adresse au public en disant que les enfants des immigrés, étant nés en Italie, se sentent "tout à fait italiens ", en soulignant que beaucoup d'entre eux parlent aussi le dialecte local (le parmesan en ce contexte).

Dans le passage qui suit (cf. T. $1 \mathrm{n}^{\circ} 3$ ), l'article aborde la proposition d'instituer des cours de dialecte dans les écoles. Un représentant de la mairie de Sant'Angelo Lodigiano (Lodi) au nord de l'Italie commente la nouvelle disant qu'il ne devrait pas y avoir de problèmes pour les élèves d'origine étrangère, en s'appuyant sur le fait que plusieurs immigrés connaissent déjà le dialecte. C'est à travers le $\mathrm{DD}$ que nous apprenons son point de vue et nous avons une indication sur sa responsabilité énonciative, dans l'incise médiane - (« conclut Crespi » le segment présentateur) qui se trouve dans l'énoncé présenté.

6. Quanto agli allievi stranieri, tra una lezione sui congiuntivi italiani e un test d'inglese dovranno misurarsi anche con il dialetto locale. «Non credo che sarà un problema - conclude Crespi -. Al mercato ogni mercoledì c' è un venditore ambulante di colore che mi saluta in dialetto santangiolino. E ha un accento invidiabile $\Perp^{31}$. (Corriere della sera, 30.08.2010)

Le politicien dans le DD raconte une anecdote concernant un vendeur ambulant qui tous les mercredis au marché lui dit bonjour en dialecte. L'énonciateur décrit le vendeur comme un homme "de couleur " qui a un accent enviable lorsqu'il parle en dialecte.

L'emploi du dialecte dans le panorama italien est généralisable aussi aux négociations entre vendeurs et clients ${ }^{32}$. De plus, les marchés sont des lieux urbains réglés par des pratiques langagières particulières. En effet, il est

31. "Quant aux élèves étrangers, entre un cours sur les subjonctifs italiens et un test d'anglais ils devront se confronter même avec le dialecte local. " "Je ne crois pas que ça sera un problème - conclut Crespi -. Au marché tous les mercredis il y a un vendeur ambulant de couleur qui me dit bonjour en dialecte de Sant'Angelo. Et il a un accent enviable. »

32. Cf. Alberto Sobrero, Sociolinguistic variables of code switching in dialectal communities in Cide-switching summer school Strasbourg, European Science Foundation, 1992, p. 17-33, cité in Anna Giacalone Ramat, "Code-switching in the context of dialect/standard language relations ", in L. Milroy, P. Muysken (sous la dir. de), One speaker two languages, Cambridge, Cambridge University Press, 1995, p. 45-67. 
important de prendre en compte la "relation d'interaction dyadique " entre client et marchand dans les pratiques communicationnelles. Ces dernières ont été décrites par le sociologue De la Pradelle ${ }^{33}$ comme le principe spécifique qui règle les échanges verbaux dans les marchés « qui les distinguent des autres formes de commercialisation, ce qui en explique la survie ou le succès ». Il souligne aussi que des pratiques linguistiques « apparemment spontanées telles qu'on les observe sur un marché sont réglées par des usages bien spécifiques (ton familier, modes d'interpellation, plaisanteries [...]) qui témoignent de la " compétence de communication " dont disposent les locuteurs" (ibidem).

Ainsi parmi les pratiques langagières typiques des marchés on retrouverait dans notre cas l'emploi du dialecte, auquel les migrants reconnaissent des qualités transactionnelles particulières qu'ils emploient souvent pour se rapprocher des natifs ${ }^{34}$.

\subsection{Dialecte : surprise et étonnement}

Lorsque des étrangers parlent un dialecte italien en présence des natifs, l'une des réactions possibles est la surprise, l'étonnement. Dans l'exemple que nous présenterons ci-dessous (cf. T. $1 \mathrm{n}^{\circ} 1$ ), tiré d'un article sur l'équipe nationale italienne de cricket, l'emploi du dialecte est présenté comme un phénomène curieux, d'autant plus qu'il est parlé à côté de l'italien, entre des joueurs d'origines différentes. C'est ce que le président de Federcricket italien, M. Gambino, remarque, comme nous le signale l'incise finale, contenant l'indication concernant l'énonciateur du segment présenté.

7. Dei tredici azzurrini (ma le squadre in campo sono composte da undici giocatori) solo uno è italiano al $100 \%$ (sardo), gli altri sono tutti figli di emigrati : due sono anglo-italiani, cinque sono bengalesi, due pakistani, due indiani e uno dello Sri Lanka. "Un pakistano e lo srilankese sono già cittadini italiani, gli altri lo saranno nei prossimi anni. E tra loro parlano più il dialetto che l'italiano ", precisa Gambino ${ }^{35}$. (Il Corriere della sera, 21.08.2009)

33. Cf. Michel De la Pradelle, "Comment d écrire un marché ", in S. Ostrowetsky S. (sous la dir. de), Sociologues en ville, Paris, L'Harmattan, 1996, p. 95.

34. Cf. Rosa Pugliese, Valeria Villa (à paraître), « Aspetti dell’integrazione linguistica degli immigrati nel contesto urbano : la percezione e l'uso dei dialetti italiani ", in G. Raimondi, L. Revelli, T. Telmon (sous la dir. de), Coesistenze Linguistiche nell'Italia Pre-e postunitaria, XLV Congresso Internazionale della Società di Linguistica Italiana, Aosta-Torino, 26-28 sett. 2011, Roma, Bulzoni.

35. Parmi les treize petits bleus (mais les équipes sur le terrain sont composées par onze joueurs) un seul est italien à $100 \%$ (sarde), les autres sont tous des fils d'émigrés : deux sont anglo-italiens, cinq sont bengalais, deux pakistanais, deux indiens et un seul du Sri Lanka. 
« Migrants et dialectes » dans la presse italienne :

représentations, enjeux et implications

Dans la représentation linguistique des joueurs, "les jeunes bleus" (gli azzurrini), comme le journaliste les appelle, nous remarquons qu'il s'agit des " enfants d'émigrés » de plusieurs pays étrangers, dont on apprend les nationalités dans le passage. Cette équipe multiculturelle est donc constituée par un seul joueur italien à $100 \%$, parmi les autres certains ont été naturalisés, d'autres le seront dans les années à venir. Mais ici l'information finale que M. Gambino tient à préciser est le partage de différents dialectes parmi les joueurs, qui selon ce qu'il affirme, de façon probablement hyperbolique, semble être plus parlé que l'italien.

Après avoir considéré un article concernant un sport peu répandu en Italie, mais surtout pratiqué par une équipe interculturelle, même l'exemple ${ }^{\circ} 8$ (cf. T.1 n $\left.{ }^{\circ} 10\right)$, d'ailleurs contenu dans la rubrique de la Repubblica «il Caso ", a de quoi étonner les lecteurs italiens. Les protagonistes de ce passage, un frère et une sœur d'origine chinoise, John et Feng Feng, ayant racheté un bar dans le centre-ville de Vérone, racontent qu'ils ont appris le dialecte véronais pour se rapprocher des clients. L'énonciateur en se servant d'un résumé avec citations, nous montre les trois règles pour le succès professionnel de deux chinois. « La gentillesse ", deuxièmement, "la qualité des produits " et enfin celle qui nous est présentée comme la plus importante « l'apprentissage du dialecte véronais ».

8. Le regole per il successo sono tre. "la gentilezza" declama John, il fratello di Feng Feng. Seconda : «la qualità dei prodotti dal caffé alle brioche ». E infine quella che sembra essere la più importante : "imparare il dialetto veronese ". Perchè i clienti vogliono sentirsi a casa anche al bar ${ }^{36}$. (La Repubblica, 18.09.2009)

L'article nous informe en outre que les gérants avaient remarqué une réticence des natifs à fréquenter un bar chinois, donc parler le dialecte peut servir pour recevoir leur approbation. En fait, selon le modèle socio-psychologique illustré dans la théorie de l'accommodation communicative de Giles ${ }^{37}$ le locuteur peut essayer de s'adapter au discours des autres, avec une stratégie de convergence, afin de réduire l'écart, la distance sociale, en mettant en place toute une série

"Un pakistanais et le sri-lankais ont déjà la nationalité italienne, les autres l'auront dans les prochaines années. Et entre eux ils parlent plus le dialecte qu'italien », précise Gambino.

36. "Les règles pour le succès sont au nombre de trois. "La gentillesse » déclame John, le frère de Feng Feng. En deuxième « la qualité des produits, du café aux brioches ». Et enfin celle qui semble être la plus importante: "apprendre le dialecte véronais ». Parce que les clients veulent se sentir chez eux, même au bar."

37. Cf. Howard Giles, Donald Taylor, Richard Bourhis, "Towards a theory of interpersonal accommodation through language : Some Canadian data " in Language in Society, 2, 1973, p. 177-192. 
de dispositifs comme l'accent, les gestes, la posture ou en s'appropriant le dialecte. En revenant aux qualités à posséder pour réussir, le responsable de cette affirmation est John, le frère de Feng Feng, comme nous le signale le segment présentateur situé à l'intérieur de l'énoncé.

Les cas $n^{\circ} 8$ et $n^{\circ} 5$ présentent un intérêt particulier car l'énonciateur est le migrant lui-même tandis qu'habituellement, dans des sujets liés à l'immigration, les intéressés sont rarement pris en compte ${ }^{38}$.

\section{Considérations conclusives}

Les passages que nous avons analysés ont mis en avant par le biais du discours direct plusieurs aspects liés à la pratique du dialecte chez les immigrés. Le DD a la fonction de nous restituer des discours authentiques sur le sujet en question et il se veut porteur de la voix de ses auteurs. Ce procédé, comme nous l'avons souligné, est désormais très fréquent dans la presse et selon la linguiste Maingueneau ${ }^{39}$ " cela peut s'expliquer à la fois par le souci de s'approcher au maximum du vécu des acteurs de la scène médiatique et par celui de sembler le plus objectif possible. Le DD répond en effet aux deux préoccupations majeures des médias : émouvoir et informer ". En même temps, à travers la procédure de mise en relief du discours rapporté ces voix - de journalistes, migrants et citoyens natifs - s'expriment sur les faits et exposent leur point de vue. Le résultat que l'on obtient très souvent est un dialogisme très immédiat qui nous montre les fonctions de la polyphonie énonciative. Il peut s'agir de souligner davantage les opinions des énonciateurs sur l'utilité du dialecte (cf. 8), sur les valeurs attribuées à la connaissance du dialecte (cf. 4, 5) et surtout de mettre en scène une image positive du migrant «italien " qui a intégré la culture et la langue locale (cf. 3, 6, 7).

Cette dernière considération peut être généralisée presque à l'ensemble des passages considérés et c'est sur cette dimension que nous nous concentrerons pour avancer des hypothèses sur les raisons de la visibilité du thème dialectemigrant dans la presse italienne. D'abord, ce type d'analyse peut être intéressant dans la mesure où il met en lumière des représentations différentes par rapport aux étiquettes habituellement réservées par les médias aux migrants. En effet, à côté d'un nombre important d'articles et de nouvelles négatives documentés par plusieurs recherches, on retrouve une petite évolution dans l'image de l'Autre, des images souvent positives qui, à travers la référence au dialecte, nous font réfléchir à l'intégration des immigrés dans le pays d'accueil. Souvent, le ton de ces articles montre une sorte de surprise, "ils parlent aussi en dialecte ", un étonnement sûrement partagé par le lecteur italien lorsque les migrants leur

38. Cf. Corte (a) op. cit. p. 2.

39. Op. cit. p. 3. 
« Migrants et dialectes » dans la presse italienne :

représentations, enjeux et implications

apparaissent plus intégrés et capables de parler la langue autrefois réservée aux natifs locaux. Il s'agit donc d'une indication supplémentaire qui paraît enrichir le portrait du migrant car elle montre une rencontre particulière et surtout insolite entre les étrangers et les langues de la tradition. Par conséquent, l'une des fonctions de ces procédés mis en œuvre par la presse pourrait être de faire naître un sentiment d'empathie chez les natifs, d'où l'intérêt probable des médias à relayer des informations de ce genre.

Même si cette nouvelle image des migrants véhiculée par la presse met l'accent sur un élément positif, la connaissance du dialecte, et non pas sur les étiquettes habituelles, cela nous amène à réfléchir ultérieurement à la représentation médiatique de l'intégration sociale. Tout d'abord la polyphonie énonciative met en scène différents acteurs qui s'expriment sur ce sujet, ce qui dans notre hypothèse se manifeste par une attention particulière accordée à d'autres voix que celle du journaliste. Il s'agit probablement d'une reconnaissance de la part des natifs des pratiques linguistiques singulières des étrangers. Enfin, un aspect nouveau se manifeste dans l'espace médiatique accordé aux immigrés : ces derniers soulignent leurs compétences dialectales qu'ils estiment être un symptôme de leur insertion sociale.

Il nous semblerait donc que ces articles ont tendance à représenter un migrant moins migrant et plus italien qui en définitive aurait acquis ce " qu'il faut savoir pour être membre" selon la définition de culture de Ward Goodenough ${ }^{40}$. Mais «membre de quoi ? Membre de la présente assemblée, membre du café à côté, de la ville de Montpellier [...] etc. ». On pourrait effectivement conclure en se demandant quelles sont les connaissances explicites et implicites (d'inclusion ou d'exclusion) qu'il faut posséder pour se sentir membre vis-àvis des autochtones ${ }^{41}$. Cependant, même cette dernière question est en partie incomplète puisqu'elle répondrait à une vision unilatérale de l'intégration. En revanche, l'enjeu majeur demeure la perspective d'une conception plus complexe et certainement bidirectionnelle de l'intégration, qui prenne en compte la coexistence de migrants et de natifs dans la reconnaissance et le respect réciproques.

40. Cf. Ward Goodenough, "Cultural anthropology and Linguistics ", Report of the Seventh Annual Round table meeting on linguistics and language study, Washington DC, Georgetown University Press, 1957, p. 167-173. Yves Winkin, "Pratique de la ville introduction à l'ethnographie urbaine " in Barberis J.-M., La ville arts de faire manières de dire, Montpellier, Paxiling, 1994, p. 99-116.

41. Yves Winkin, "Pratique de la ville introduction à l'ethnographie urbaine ", in Barberis J.-M., La ville arts de faire manières de dire, op. cit., p. 101. 